\title{
Right Sided Ideals and Multilinear Polynomials with Derivations on Prime Rings
}

\author{
Basudeb Dhara (*) - RaJendra K. Sharma (**)
}

ABstRACT - Let $R$ be an associative prime ring of char $R \neq 2$ with center $Z(R)$ and extended centroid $C, f\left(x_{1}, \ldots, x_{n}\right)$ a nonzero multilinear polynomial over $C$ in $n$ noncommuting variables, $d$ a nonzero derivation of $R$ and $\rho$ a nonzero right ideal of $R$. We prove that: (i) if $\left[d^{2}\left(f\left(x_{1}, \ldots, x_{n}\right)\right), f\left(x_{1}, \ldots, x_{n}\right)\right]=0$ for all $x_{1}, \ldots, x_{n} \in \rho$ then $\rho C=e R C$ for some idempotent element $e$ in the socle of $R C$ and $f\left(x_{1}, \ldots, x_{n}\right)$ is central-valued in $e R C e$ unless $d$ is an inner derivation induced by $b \in Q$ such that $b^{2}=0$ and $b \rho=0$; (ii) if $\left[d^{2}\left(f\left(x_{1}, \ldots, x_{n}\right)\right), f\left(x_{1}, \ldots, x_{n}\right)\right] \in Z(R)$ for all $x_{1}, \ldots, x_{n} \in \rho$ then $\rho C=e R C$ for some idempotent element $e$ in the socle of $R C$ and either $f\left(x_{1}, \ldots, x_{n}\right)$ is central in $e R C e$ or $e R C e$ satisfies the standard identity $S_{4}\left(x_{1}, x_{2}, x_{3}, x_{4}\right)$ unless $d$ is an inner derivation induced by $b \in Q$ such that $b^{2}=0$ and $b \rho=0$.

Throughout this paper, $R$ always denotes a prime ring with extended centroid $C$ and $Q$ its two-sided Martindale ring of quotient. By $d$ we mean a nonzero derivation of $R$. For $x, y \in R$, the commutator of $x, y$ is denoted by $[x, y]$ and defined by $[x, y]=x y-y x$. We denote $[x, y]_{2}=[[x, y], y]=$ $=[x, y] y-y[x, y]$.

A well known result proved by Posner [17] states that $R$ must be commutative if $[d(x), x] \in Z(R)$ for all $x \in R$. In [10] Lanski generalized the Posner's result to a Lie ideal. More precisely Lanski proved that if $L$ is a noncommutative Lie ideal of $R$ such that $[d(x), x] \in Z(R)$ for all $x \in L$,

(*) Indirizzo dell'A.: Department of Mathematics, Belda College, Belda, Paschim Medinipur-721424, India.

E-mail: basu_dhara@yahoo.com

(**) Indirizzo dell'A.: Department of Mathematics, Indian Institute of Technology, Delhi, Hauz Khas, New Delhi-110016, India.

E-mail: rksharma@maths.iitd.ac.in

Mathematics Subject Classification: 16W25, 16R50, 16N60.

This work is supported by a grant from University Grants Commission, India. 
then char $R=2$ and $R$ satisfies $S_{4}\left(x_{1}, x_{2}, x_{3}, x_{4}\right)$, the standard identity. Note that a noncommutative Lie ideal of $R$ contains all the commutators [ $\left.x_{1}, x_{2}\right]$ for $x_{1}, x_{2}$ in some nonzero ideal of $R$ ( see [10, Lemma 2 (i), (ii)]). So, it is natural to consider the situation when $[d(x), x] \in Z(R)$ for all commutators $x=\left[x_{1}, x_{2}\right]$ or more general case $x=f\left(x_{1}, \ldots, x_{n}\right)$ where $f\left(x_{1}, \ldots, x_{n}\right)$ is a multilinear polynomial. In [11] Lee and Lee proved that if $\left[d\left(f\left(x_{1}, \ldots, x_{n}\right)\right), f\left(x_{1}, \ldots, x_{n}\right)\right] \in Z(R)$ for all $x_{1}, \ldots, x_{n}$ in some nonzero ideal of $R$, then $f\left(x_{1}, \ldots, x_{n}\right)$ is central-valued on $R$, except when char $R=2$ and $R$ satisfies $S_{4}\left(x_{1}, x_{2}, x_{3}, x_{4}\right)$. Recently, De Filippis and Di Vincenzo (see [7]) consider the situation $\delta\left(\left[d\left(f\left(x_{1}, \ldots, x_{n}\right)\right), f\left(x_{1}, \ldots, x_{n}\right)\right]\right)=0$ for all $x_{1}, \ldots, x_{n} \in R$, where $d$ and $\delta$ are two derivations of $R$. The statement of De Filippis and Di Vincenzo's theorem is the following:

THEOREM A ([7, Theorem 1]). Let $K$ be a noncommutative ring with unity, $R$ a prime $K$-algebra of characteristic different from $2, d$ and $\delta$ nonzero derivations of $R$ and $f\left(x_{1}, \ldots, x_{n}\right)$ a multilinear polynomial over $K$. If $\delta\left(\left[d\left(f\left(x_{1}, \ldots, x_{n}\right)\right), f\left(x_{1}, \ldots, x_{n}\right)\right]\right)=0$ for all $x_{1}, \ldots, x_{n} \in R$, then $f\left(x_{1}, \ldots, x_{n}\right)$ is central-valued on $R$.

In case $\delta$ and $d$ are two same derivations, the differential identity becomes $\left[d^{2}\left(f\left(x_{1}, \ldots, x_{n}\right)\right), f\left(x_{1}, \ldots, x_{n}\right)\right]=0$ for all $x_{1}, \ldots, x_{n} \in R$. So, it is natural to ask, what happen in cases $\left[d^{2}\left(f\left(x_{1}, \ldots, x_{n}\right)\right), f\left(x_{1}, \ldots, x_{n}\right)\right] \in Z(R)$ for all $x_{1}, \ldots, x_{n} \in R$ and $\left[d^{2}\left(f\left(x_{1}, \ldots, x_{n}\right)\right), f\left(x_{1}, \ldots, x_{n}\right)\right] \in Z(R)$ for all $x_{1}, \ldots, x_{n} \in \rho$, where $\rho$ is a non-zero right ideal of $R$. In the present paper our object is to study these cases.

For the sake of completeness we recall some basic notations, definitions and some easy consequences of the result of Kharchenko [8] about the differential identities on a prime ring $R$. First, we denote by $\operatorname{Der}(Q)$ the set of all derivations on $Q$. By a derivation word $\Delta$ of $R$ we mean $\Delta=d_{1} d_{2} d_{3} \ldots d_{m}$ for some derivations $d_{i}$ of $R$. For $x \in R$, we denote by $x^{\Delta}$ the image of $x$ under $\Delta$, that is $x^{\Delta}=\left(\cdots\left(x^{d_{1}}\right)^{d_{2}} \cdots\right)^{d_{m}}$. By a differential polynomial, we mean a generalized polynomial, with coefficients in $Q$, of the form $\Phi\left(x_{i}^{U_{j}}\right)$ involving noncommutative indeterminates $x_{i}$ on which the derivations words $\Delta_{j}$ act as unary operations. $\Phi\left(x_{i}^{\Delta_{j}}\right)=0$ is said to be a differential identity on a subset $T$ of $Q$ if it vanishes for any assignment of values from $T$ to its indeterminates $x_{i}$.

Now let $D_{\text {int }}$ be the $C$-subspace of $\operatorname{Der}(Q)$ consisting of all inner derivations on $Q$. By Kharchenko's theorem [8, Theorem 2], we have the following result: 
Let $R$ be a prime ring of characteristic different from 2 . If two nonzero derivations $d$ and $\delta$ are $C$-linearly independent modulo $D_{\text {int }}$ and $\Phi\left(x_{i}^{\Delta_{j}}\right)$ is a differential identity on $R$, where $\Delta_{j}$ are derivations words of the following form $\delta, d, \delta^{2}, \delta d, d^{2}$, then $\Phi\left(y_{j i}\right)$ is a generalized polynomial identity on $R$, where $y_{j i}$ are distinct indeterminates.

As a particular case, we have:

If $d$ is a nonzero derivation on $R$ and $\Phi\left(x_{1}, \ldots, x_{n}, x_{1}^{d}, \ldots, x_{n}^{d}\right.$, $\left.x_{1}^{d^{2}}, \ldots, x_{n}^{d^{2}}\right)$ is a differential identity on $R$, then one of the following holds:

(i) either $d \in D_{\text {int }}$

Or

(ii) $R$ satisfies the generalized polynomial identity $\Phi\left(x_{1}, \ldots, x_{n}\right.$, $\left.y_{1}, \ldots, y_{n}, z_{1}, \ldots, z_{n}\right)$

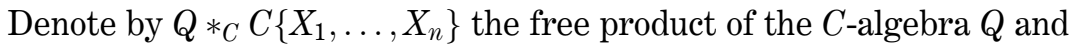
$C\left\{X_{1}, \ldots, X_{n}\right\}$, the free $C$-algebra in noncommuting indeterminates $X_{1}, \ldots, X_{n}$.

Since $f\left(x_{1}, \ldots, x_{n}\right)$ is a multilinear polynomial, we can write

$$
f\left(x_{1}, \ldots, x_{n}\right)=x_{1} x_{2} \ldots x_{n}+\sum_{I \neq \sigma \in S_{n}} \alpha_{\sigma} x_{\sigma(1)} \ldots x_{\sigma(n)}
$$

where $S_{n}$ is the permutation group over $n$ elements and any $\alpha_{\sigma} \in C$. We denote by $f^{d}\left(x_{1}, \ldots, x_{n}\right)$ the polynomial obtained from $f\left(x_{1}, \ldots, x_{n}\right)$ by replacing each coefficient $\alpha_{\sigma}$ with $d\left(\alpha_{\sigma} .1\right)$. In this way we have

$$
d\left(f\left(x_{1}, \ldots, x_{n}\right)\right)=f^{d}\left(x_{1}, \ldots, x_{n}\right)+\sum_{i} f\left(x_{1}, \ldots, d\left(x_{i}\right), \ldots, x_{n}\right)
$$

and

$$
\begin{gathered}
d^{2}\left(f\left(x_{1}, \ldots, x_{n}\right)\right)=d\left(f^{d}\left(x_{1}, \ldots, x_{n}\right)\right)+d\left(\sum_{i} f\left(x_{1}, \ldots, d\left(x_{i}\right), \ldots, x_{n}\right)\right) \\
=f^{d^{2}}\left(x_{1}, \ldots, x_{n}\right)+\sum_{i} f^{d}\left(x_{1}, \ldots, d\left(x_{i}\right), \ldots, x_{n}\right) \\
+\sum_{i} f^{d}\left(x_{1}, \ldots, d\left(x_{i}\right), \ldots, x_{n}\right)+\sum_{i \neq j} f\left(x_{1}, \ldots, d\left(x_{i}\right), \ldots, d\left(x_{j}\right), \ldots, x_{n}\right) \\
+\sum_{i} f\left(x_{1}, \ldots, d^{2}\left(x_{i}\right), \ldots, x_{n}\right) \\
=f^{d^{2}}\left(x_{1}, \ldots, x_{n}\right)+2 \sum_{i} f^{d}\left(x_{1}, \ldots, d\left(x_{i}\right), \ldots, x_{n}\right) \\
+2 \sum_{i<j} f\left(x_{1}, \ldots, d\left(x_{i}\right), \ldots, d\left(x_{j}\right), \ldots, x_{n}\right)+\sum_{i} f\left(x_{1}, \ldots, d^{2}\left(x_{i}\right), \ldots, x_{n}\right) .
\end{gathered}
$$




\section{The case for $\rho=R$.}

LEMMA 1.1. Let $R=M_{k}(F)$ be the ring of all $k \times k$ matrices over a field $F$ of characteristic $\neq 2, b \in R$ and $f\left(x_{1}, \ldots, x_{n}\right)$ is a multilinear polynomial over $F$. If $k \geq 2$ and $\left[\left[b,\left[b, f\left(x_{1}, \ldots, x_{n}\right)\right]\right], f\left(x_{1}, \ldots, x_{n}\right)\right]=0$ for all $x_{1}, \ldots, x_{n} \in R$ or if $k \geq 3$ and $\left[\left[b,\left[b, f\left(x_{1}, \ldots, x_{n}\right)\right]\right], f\left(x_{1}, \ldots, x_{n}\right)\right] \in Z(R)$ for all $x_{1}, \ldots, x_{n} \in R$, then either $b \in F \cdot I_{k}$ or $f\left(x_{1}, \ldots, x_{n}\right)$ is central-valued on $R$.

Proof. Let $b=\left(b_{i j}\right)_{k \times k}$. Let $e_{i j}$ be the usual matrix unit with 1 in $(i, j)$ entry and zero else where. Now we proceed to show that $b \in Z(R)$ if $\in f\left(x_{1}, \ldots, x_{n}\right)$ is non central valued on $R$.

For simplicity, we write $f\left(x_{1}, \ldots, x_{n}\right)=f(x)$, where $x=\left(x_{1}, \ldots, x_{n}\right)$ $R^{n}=R \times \cdots \times R$ ( $n$ times). Then by assumption,

$$
[[b,[b, f(x)]], f(x)]=\left[b^{2} f(x)-2 b f(x) b+f(x) b^{2}, f(x)\right] \in Z(R)
$$

for all $x \in R^{n}$. Since $f\left(x_{1}, \ldots, x_{n}\right)$ is assumed to be noncentral on $R$, by [15, Lemma 2, Proof of Lemma 3] there exists a sequence of matrices $r=\left(r_{1}, \ldots, r_{n}\right)$ in $R$ such that $f(r)=f\left(r_{1}, \ldots, r_{n}\right)=\alpha e_{i j} \neq 0$ where $0 \neq \alpha \in F$ and $i \neq j$. Thus

$$
\left[b^{2} \alpha e_{i j}-2 b \alpha e_{i j} b+\alpha e_{i j} b^{2}, \alpha e_{i j}\right] \in Z(R) .
$$

Since the rank of $\left[b^{2} \alpha e_{i j}-2 b \alpha e_{i j} b+\alpha e_{i j} b^{2}, \alpha e_{i j}\right]$ is $\leq 2,\left[b^{2} \alpha e_{i j}-2 b \alpha e_{i j} b+\right.$ $\left.+\alpha e_{i j} b^{2}, \alpha e_{i j}\right]=0$. Left multiplying by $e_{i j}$, we get $0=e_{i j}\left(-2 b \alpha e_{i j} b \alpha e_{i j}\right)=$ $=-2 \alpha^{2} b_{j i}^{2} e_{i j}$. Since char $F \neq 2, b_{j i}=0$. For $s \neq t$, let $\sigma$ be a permutation in the symmetric group $S_{m}$ such that $\sigma(i)=s$ and $\sigma(j)=t$. Let $\psi$ be the automorphism of $R$ defined by $x^{\psi /}=\left(\sum_{p, q} \xi_{p q} e_{p q}\right)^{\psi}=\sum_{p, q} \xi_{p q} e_{\sigma(p), \sigma(q)}$. Then $f\left(r^{\psi /}\right)=f\left(r_{1}^{\psi}, \ldots, r_{n}^{\psi}\right)=f(r)^{\psi /}=\alpha e_{s t} \neq 0$ and we have as above $b_{t s}=0$ for $s \neq t$. Thus $b$ is a diagonal matrix. For any $F$-automorphism $\theta$ of $R, b^{\theta}$ enjoys the same property as $b$ does, namely, $\left[\left[b^{\theta},\left[b^{\theta}, f(x)\right]\right], f(x)\right] \in Z(R)$ for all $x \in R^{n}$. Hence, $b^{\theta}$ must be diagonal. Write $b=\sum_{i=1}^{k} a_{i i} e_{i i}$; then for each $j \neq 1$,
we have

$$
\left(1+e_{1 j}\right) b\left(1-e_{1 j}\right)=\sum_{i=1}^{k} a_{i i} e_{i i}+\left(b_{j j}-b_{11}\right) e_{1 j}
$$

diagonal. Therefore, $b_{j j}=b_{11}$ and so $b$ is a scalar matrix. 
LEMMA 1.2. Let $R$ be a prime ring of characteristic different from 2 and $f\left(x_{1}, \ldots, x_{n}\right)$ a multilinear polynomial over $C$. If for any $i=1, \ldots, n$,

$$
\left[f\left(x_{1}, \ldots, z_{i}, \ldots, x_{n}\right), f\left(x_{1}, \ldots, x_{n}\right)\right]=0
$$

for all $x_{1}, \ldots, x_{n}, z_{i} \in R$, then the polynomialf $\left(x_{1}, \ldots, x_{n}\right)$ is central-valued on $R$.

Proof. Let $a$ be a noncentral element of $R$. Then replacing $z_{i}$ with $\left[a, x_{i}\right]$ we have that for any $i=1, \ldots, n$

$$
\left[f\left(x_{1}, \ldots,\left[a, x_{i}\right], \ldots, x_{n}\right), f\left(x_{1}, \ldots, x_{n}\right)\right]=0
$$

and so

$$
\left[\sum_{i=0}^{n} f\left(x_{1}, \ldots,\left[a, x_{i}\right], \ldots, x_{n}\right), f\left(x_{1}, \ldots, x_{n}\right)\right]=0
$$

which implies, $\left[a, f\left(x_{1}, \ldots, x_{n}\right)\right]_{2}=0$ for all $x_{1}, \ldots, x_{n} \in R$. By [11, Theorem $]$, $f\left(x_{1}, \ldots, x_{n}\right)$ is central-valued on $R$.

THEOREM 1.3. Let $R$ be a prime ring of characteristic different from $2, d$ a nonzero derivation of $R, f\left(x_{1}, \ldots, x_{n}\right)$ a multilinear polynomial over $C$. If

$$
\left[d^{2}\left(f\left(x_{1}, \ldots, x_{n}\right)\right), f\left(x_{1}, \ldots, x_{n}\right)\right] \in Z(R) \text { for all } x_{1}, \ldots, x_{n} \in R
$$

then either $f\left(x_{1}, \ldots, x_{n}\right)$ is central-valued on $R$ or $R$ satisfies the standard identity $S_{4}\left(x_{1}, x_{2}, x_{3}, x_{4}\right)$.

Proof. Let $I$ be any nonzero two-sided ideal of $R$. If for every $r_{1}, \ldots, r_{n} \in I, \quad\left[d^{2}\left(f\left(r_{1}, \ldots, r_{n}\right)\right), f\left(r_{1}, \ldots, r_{n}\right)\right]=0$, then by [14], this generalized differential identity is also satisfied by $Q$ and hence by $R$ as well. By Theorem $A, f\left(r_{1}, \ldots, r_{n}\right)$ is then central-valued on $R$ and we are done. Now we assume that for some $r_{1}, \ldots, r_{n} \in I$, $0 \neq\left[d^{2}\left(f\left(r_{1}, \ldots, r_{n}\right)\right), f\left(r_{1}, \ldots, r_{n}\right)\right] \in I \cap Z(R)$. Thus $I \cap Z(R) \neq 0$. Let $K$ be a nonzero two-sided ideal of $R_{Z}$, the ring of central quotients of $R$. Since $K \cap R$ is a nonzero two-sided ideal of $R,(K \cap R) \cap Z(R) \neq 0$. Therefore, $K$ contains an invertible element in $R_{Z}$ and so $R_{Z}$ is a simple ring with identity 1. 
By assumption, $R$ satisfies the differential identity

$$
\begin{aligned}
& g\left(x_{1}, \ldots, x_{n}, d\left(x_{1}\right), \ldots, d\left(x_{n}\right), d^{2}\left(x_{1}\right), \ldots, d^{2}\left(x_{n}\right)\right) \\
& =\left[\left[f^{d^{2}}\left(x_{1}, \ldots, x_{n}\right)+2 \sum_{i} f^{d}\left(x_{1}, \ldots, d\left(x_{i}\right), \ldots, x_{n}\right)\right.\right. \\
& \quad+2 \sum_{i<j} f\left(x_{1}, \ldots, d\left(x_{i}\right), \ldots, d\left(x_{j}\right), \ldots, x_{n}\right) \\
& \left.\left.+\sum_{i} f\left(x_{1}, \ldots, d^{2}\left(x_{i}\right), \ldots, x_{n}\right), f\left(x_{1}, \ldots, x_{n}\right)\right], x_{n+1}\right] .
\end{aligned}
$$

If $d$ is not $Q$-inner, then by Kharchenko's theorem [8],

$$
\begin{aligned}
& {\left[\left[f^{d^{2}}\left(x_{1}, \ldots, x_{n}\right)+2 \sum_{i} f^{d}\left(x_{1}, \ldots, y_{i}, \ldots, x_{n}\right)\right.\right.} \\
& +2 \sum_{i<j} f\left(x_{1}, \ldots, y_{i}, \ldots, y_{j}, \ldots, x_{n}\right) \\
& \left.\left.+\sum_{i} f\left(x_{1}, \ldots, z_{i}, \ldots, x_{n}\right), f\left(x_{1}, \ldots, x_{n}\right)\right], x_{n+1}\right]=0
\end{aligned}
$$

for all $x_{i}, y_{i}, z_{i}, x_{n+1} \in R$ for $i=1,2, \ldots, n$. In particular, for any $i$, assuming $y_{1}=\cdots=y_{i-1}=y_{i+1}=\cdots=y_{n}=0, z_{1}=\cdots=z_{n}=0$, we have

$$
\left[\left[f^{d^{2}}\left(x_{1}, \ldots, x_{n}\right)+2 f^{d}\left(x_{1}, \ldots, y_{i}, \ldots, x_{n}\right), f\left(x_{1}, \ldots, x_{n}\right)\right], x_{n+1}\right]=0
$$

and so

$$
\left[\left[f^{d^{2}}\left(x_{1}, \ldots, x_{n}\right)+2 \sum_{i} f^{d}\left(x_{1}, \ldots, y_{i}, \ldots, x_{n}\right), f\left(x_{1}, \ldots, x_{n}\right)\right], x_{n+1}\right]=0
$$

for all $x_{i}, y_{i}, x_{n+1} \in R, i=1,2, \ldots, n$. Thus from (1), we obtain

$$
\begin{gathered}
{\left[\left[2 \sum_{i<j} f\left(x_{1}, \ldots, y_{i}, \ldots, y_{j}, \ldots, x_{n}\right)\right.\right.} \\
\left.\left.+\sum_{i} f\left(x_{1}, \ldots, z_{i}, \ldots, x_{n}\right), f\left(x_{1}, \ldots, x_{n}\right)\right], x_{n+1}\right]=0
\end{gathered}
$$

for all $x_{i}, y_{i}, z_{i}, x_{n+1} \in R$ for $i=1,2, \ldots, n$.

By localizing $R$ at $Z(R)$, we obtain that (2) is also an identity of $R_{Z}$. Since $R$ and $R_{Z}$ satisfy the same polynomial identities, in order to prove that $R$ satisfies $S_{4}$, we may assume that $R$ is a simple ring with 1 . Thus $R$ satisfies the identity (2). Now putting $y_{i}=\left[b, x_{i}\right]=\delta\left(x_{i}\right)$ and $z_{i}=\left[b,\left[b, x_{i}\right]\right]=$ 
$=\delta^{2}\left(x_{i}\right), i=1,2, \ldots, n$ for some $b \notin Z(R)$, where $\delta$ is an inner derivation induced by some $b \in R$, we obtain that $R$ satisfies

$$
\left[\left[\delta^{2}\left(f\left(x_{1}, \ldots, x_{n}\right)\right), f\left(x_{1}, \ldots, x_{n}\right)\right], x_{n+1}\right]=0 .
$$

Thus by Martindale's theorem [16], $R$ is a primitive ring with a minimal right ideal, whose commuting ring $D$ is a division ring which is finite dimensional over $Z(R)$. However, since $R$ is simple with $1, R$ must be Artinian. Hence $R=D_{k^{\prime}}$, the ring of $k^{\prime} \times k^{\prime}$ matrices over $D$, for some $k^{\prime} \geq 1$. Again, by [9, Lemma 2], it follows that there exists a field $F$ such that $R \subseteq M_{k}(F)$, the ring of all $k \times k$ matrices over the field $F$, and $M_{k}(F)$ satisfies

$$
\left[\left[\delta^{2}\left(f\left(x_{1}, \ldots, x_{n}\right)\right), f\left(x_{1}, \ldots, x_{n}\right)\right], x_{n+1}\right]=0 .
$$

If $k \geq 3$, then by Lemma 1.1, we have $b \in Z(R)$, a contradiction. Thus $k=2$, that is, $R$ satisfies $S_{4}\left(x_{1}, x_{2}, x_{3}, x_{4}\right)$.

Similarly, the same conclusion can be drawn in case $d$ is an $Q$-inner derivation induced by some $b \in Q$.

\section{The case for one-sided ideal.}

We begin with the following lemmas

Lemma 2.1. Let $\rho$ be a nonzero right ideal of $R$ and $d$ a derivation of $R$. Then the following conditions are equivalent:

(i) $d$ is an inner derivation induced by some $b \in Q$ such that $b \rho=0$;

(ii) $d(\rho) \rho=0$.

For its proof, we refer to [2, Lemma].

Lemma 2.2. Let $R$ be a prime ring, $\rho$ a nonzero right ideal of $R$, $f\left(x_{1}, \ldots, x_{t}\right)$ a multilinear polynomial over $C, a \in R$ and $n$ a fixed positive integer. If $f\left(x_{1}, \ldots, x_{t}\right)^{n} a=0$ for all $x_{1}, \ldots, x_{t} \in \rho$, then either $a=0$ or $f(\rho) \rho=0$.

For its proof, we refer to [3, Lemma 2 (II)].

LEMMA 2.3. Let $R$ be a prime ring. If $\left[d^{2}\left(f\left(x_{1}, \ldots, x_{n}\right)\right), f\left(x_{1}, \ldots, x_{n}\right)\right] \in$ $Z(R)$ for all $x_{1}, \ldots, x_{n} \in \rho$, then $R$ satisfies nontrivial generalized polynomial identity unless $d$ is an inner derivation induced by $b \in Q$ such that $b^{2}=0$ and $b \rho=0$. 
Proof. Suppose on the contrary that $R$ does not satisfy any nontrivial generalized polynomial identity (GPI). Thus we may assume that $R$ is noncommutative, otherwise $R$ satisfies trivially a nontrivial GPI. Now we consider the following two cases:

CASE I. Suppose that $d$ is a $Q$-inner derivation induced by an element $b \in Q$ such that $b^{2} \neq 0$. Then for any $x_{0} \in \rho$

$$
\left[\left[b,\left[b, f\left(x_{0} X_{1}, \ldots, x_{0} X_{n}\right)\right]\right], f\left(x_{0} X_{1}, \ldots, x_{0} X_{n}\right)\right] \in Z(R)
$$

that is

$$
\begin{array}{r}
{\left[\left[b^{2} f\left(x_{0} X_{1}, \ldots, x_{0} X_{n}\right)-2 b f\left(x_{0} X_{1}, \ldots, x_{0} X_{n}\right) b\right.\right.} \\
\left.\left.+f\left(x_{0} X_{1}, \ldots, x_{0} X_{n}\right) b^{2}, f\left(x_{0} X_{1}, \ldots, x_{0} X_{n}\right)\right], x_{0} X_{n+1}\right]
\end{array}
$$

is a GPI for $R$, so it is the zero element in $Q *_{C} C\left\{X_{1}, \ldots, X_{n+1}\right\}$. Denote $l_{R}(\rho)$ the left annihilator of $\rho$ in $R$. Suppose first that $\left\{1, b, b^{2}\right\}$ are linearly $C$-independent modulo $l_{R}(\rho)$, that is $\left(\alpha b^{2}+\beta b+\gamma\right) \rho=0$ if and only if $\alpha=\beta=\gamma=0$. Since $R$ is not a GPI-ring, a fortiori it can not be a PI-ring. Thus, by [13, Lemma 3] there exists $x_{0} \in \rho$ such that $\left\{b^{2} x_{0}, b x_{0}, x_{0}\right\}$ are linearly $C$-independent. Then we have that

$$
\begin{gathered}
{\left[\left[b^{2} f\left(x_{0} X_{1}, \ldots, x_{0} X_{n}\right)-2 b f\left(x_{0} X_{1}, \ldots, x_{0} X_{n}\right) b\right.\right.} \\
\left.\left.+f\left(x_{0} X_{1}, \ldots, x_{0} X_{n}\right) b^{2}, f\left(x_{0} X_{1}, \ldots, x_{0} X_{n}\right)\right], x_{0} X_{n+1}\right]=0
\end{gathered}
$$

is a nontrivial GPI for $R$, a contradiction.

Therefore, $\left\{1, b, b^{2}\right\}$ are linearly $C$-dependent modulo $l_{R}(\rho)$, that is there exist $\alpha, \beta, \gamma \in C$, not all zero, such that $\left(\alpha b^{2}+\beta b+\gamma\right) \rho=0$. Suppose that $\alpha=0$. Then $\beta \neq 0$, otherwise $\gamma=0$. Thus by $(\beta b+\gamma) \rho=0$, we have that $\left(b+\beta^{-1} \gamma\right) \rho=0$. Since $b$ and $b+\beta^{-1} \gamma$ induce the same inner derivation, we may replace $b$ by $b+\beta^{-1} \gamma$ in the basic hypothesis. Therefore, in any case we may suppose $b \rho=0$ and then from (3), $R$ satisfies $x_{0} X_{n+1} f^{2}\left(x_{0} X_{1}, \ldots, x_{0} X_{n}\right) b^{2}=0$. Since $R$ does not satisfy any nontrivial GPI, $b^{2}=0$, a contradiction.

Next suppose that $\alpha \neq 0$. In this case there exist $\lambda, \mu \in C$ such that $b^{2} x_{0}=\lambda b x_{0}+\mu x_{0}$ for all $x_{0} \in \rho$. If $b x_{0}$ and $x_{0}$ are linearly $C$ dependent for all $x_{0} \in \rho$, then again we obtain $b \rho=0$ and so $b^{2}=0$. Therefore choose $x_{0} \in \rho$ such that $b x_{0}$ and $x_{0}$ are linearly $C$-independent. Then replacing $b^{2} x_{0}$ with $\lambda b x_{0}+\mu x_{0}$, we obtain from (3) 
that $R$ satisfies

$$
\begin{aligned}
& {\left[\left\{(\lambda b+\mu) f^{2}\left(x_{0} X_{1}, \ldots, x_{0} X_{n}\right)-2 b f\left(x_{0} X_{1}, \ldots, x_{0} X_{n}\right) b f\left(x_{0} X_{1}, \ldots, x_{0} X_{n}\right)\right.\right.} \\
& \left.\quad+f\left(x_{0} X_{1}, \ldots, x_{0} X_{n}\right)(\lambda b+\mu) f\left(x_{0} X_{1}, \ldots, x_{0} X_{n}\right)\right\} \\
& \quad-\left\{f\left(x_{0} X_{1}, \ldots, x_{0} X_{n}\right)(\lambda b+\mu) f\left(x_{0} X_{1}, \ldots, x_{0} X_{n}\right)\right. \\
& \left.\left.-2 f\left(x_{0} X_{1}, \ldots, x_{0} X_{n}\right) b f\left(x_{0} X_{1}, \ldots, x_{0} X_{n}\right) b+f^{2}\left(x_{0} X_{1}, \ldots, x_{0} X_{n}\right) b^{2}\right\}, x_{0} X_{n+1}\right] .
\end{aligned}
$$

This is a nontrivial GPI for $R$, because the term

$$
\left(\lambda b f^{2}\left(x_{0} X_{1}, \ldots, x_{0} X_{n}\right)-2 b f\left(x_{0} X_{1}, \ldots, x_{0} X_{n}\right) b f\left(x_{0} X_{1}, \ldots, x_{0} X_{n}\right)\right) x_{0} X_{n+1}
$$

appears nontrivially, a contradiction.

CASE II. Suppose that $d$ is an inner derivation induced by an element $b \in Q$ such that $b^{2}=0$. Thus we have that $\left[-2 b f\left(X_{1}, \ldots, X_{n}\right) b, f\left(X_{1}, \ldots, X_{n}\right)\right] \in$ $Z(R)$ is satisfied by $\rho$. In case there exists $x_{0} \in \rho$ such that $\left\{b x_{0}, x_{0}\right\}$ are linearly $C$-independent, we have that $\left[\left[-2 b f\left(x_{0} X_{1}, \ldots, x_{0} X_{n}\right) b\right.\right.$, $\left.\left.f\left(x_{0} X_{1}, \ldots, x_{0} X_{n}\right)\right], x_{0} X_{n+1}\right]$ is a non trivial GPI for $R$, a contradiction. Hence $\left\{b x_{0}, x_{0}\right\}$ are linearly $C$-dependent for all $x_{0} \in \rho$, that is there exists $\alpha \in C$ such that $(b-\alpha) \rho=0$. Thus we have that $\left[\alpha f^{2}\left(X_{1}, \ldots, X_{n}\right)(\alpha-b), X_{n+1}\right]$ is satisfied by $\rho$, in particular $R$ satisfies:

$$
\left[\alpha f^{2}\left(x_{0} X_{1}, \ldots, x_{0} X_{n}\right)(\alpha-b), f\left(x_{0} X_{1}, \ldots, x_{0} X_{n}\right)\right]=\alpha f^{3}\left(X_{1}, \ldots, X_{n}\right)(\alpha-b)
$$

for any $x_{0} \in \rho$. Since $R$ is not GPI, it follows that either $b=\alpha \in C$, which is a contradiction, or $\alpha=0$ which means $b \rho=0$, as required.

CASE III. Suppose that $d$ is an inner derivation induced by an element $b \in Q$ such that $b \rho=0$. Thus we have that $\left[-f^{2}\left(X_{1}, \ldots, X_{n}\right) b^{2}, X_{n+1}\right]$ is satisfied by $\rho$, in particular $R$ satisfies:

$$
\left[-f^{2}\left(x_{0} X_{1}, \ldots, x_{0} X_{n}\right) b^{2}, f\left(x_{0} X_{1}, \ldots, x_{0} X_{n}\right)\right]=f^{3}\left(x_{0} X_{1}, \ldots, x_{0} X_{n}\right) b^{2}
$$

for any $x_{0} \in \rho$. Again since $R$ is not GPI we conclude that $b^{2}=0$.

CAse IV. Next suppose that $d$ is not $Q$-inner derivation. By our assumption we have that $R$ satisfies

$$
\begin{gathered}
0=\left[\left[f^{d^{2}}\left(x X_{1}, \ldots, x X_{n}\right)+2 \sum_{i} f^{d}\left(x X_{1}, \ldots, d(x) X_{i}+x d\left(X_{i}\right), \ldots, x X_{n}\right)\right.\right. \\
+2 \sum_{i<j} f\left(x X_{1}, \ldots, d(x) X_{i}+x d\left(X_{i}\right), \ldots, d(x) X_{j}+x d\left(X_{j}\right), \ldots, x X_{n}\right) \\
\left.\left.+\sum_{i} f\left(x X_{1}, \ldots, d^{2}(x) X_{i}+2 d(x) d\left(X_{i}\right)+x d^{2}\left(X_{i}\right), \ldots, x X_{n}\right), f\left(x X_{1}, \ldots, x X_{n}\right)\right], X_{n+1}\right] .
\end{gathered}
$$


By Kharchenko's theorem [8],

$$
\begin{gathered}
{\left[\left[f^{d^{2}}\left(x X_{1}, \ldots, x X_{n}\right)+2 \sum_{i} f^{d}\left(x X_{1}, \ldots, d(x) X_{i}+x r_{i}, \ldots, x X_{n}\right)\right.\right.} \\
+2 \sum_{i<j} f\left(x X_{1}, \ldots, d(x) X_{i}+x r_{i}, \ldots, d(x) X_{j}+x r_{j}, \ldots, x X_{n}\right) \\
\left.\left.+\sum_{i} f\left(x X_{1}, \ldots, d^{2}(x) X_{i}+2 d(x) r_{i}+x s_{i}, \ldots, x X_{n}\right), f\left(x X_{1}, \ldots, x X_{n}\right)\right], X_{n+1}\right]=0
\end{gathered}
$$

for all $X_{1}, \ldots, X_{n}, r_{1}, \ldots, r_{n}, s_{1}, \ldots, s_{n} \in R$. In particular, for $r_{1}=$ $=r_{2}=\cdots=r_{n}=0$, we have

$$
\begin{gathered}
{\left[\left[f^{d^{2}}\left(x X_{1}, \ldots, x X_{n}\right)+2 \sum_{i} f^{d}\left(x X_{1}, \ldots, d(x) X_{i}, \ldots, x X_{n}\right)\right.\right.} \\
2 \sum_{i<j} f\left(x X_{1}, \ldots, d(x) X_{i}, \ldots, d(x) X_{j}, \ldots, x X_{n}\right)+\sum_{i} f\left(x X_{1}, \ldots, d^{2}(x) X_{i}, \ldots, x X_{n}\right)+ \\
\left.\left.+\sum_{i} f\left(x X_{1}, \ldots, x s_{i}, \ldots, x X_{n}\right), f\left(x X_{1}, \ldots, x X_{n}\right)\right], X_{n+1}\right]=0 .
\end{gathered}
$$

Hence $R$ satisfies the blended component

$$
\left[\left[f\left(x s_{1}, \ldots, x X_{n}\right), f\left(x X_{1}, \ldots, x X_{n}\right)\right], X_{n+1}\right]=0
$$

which is a nontrivial GPI for $R$, a contradiction.

THEOREM 2.4. Let $R$ be an associative prime ring of char $R \neq 2$ with center $Z(R)$ and extended centroid $C, f\left(x_{1}, \ldots, x_{n}\right)$ a nonzero multilinear polynomial over $C$ in n noncommuting variables, d a nonzero derivation of $R$ and $\rho$ a nonzero right ideal of $R$. If $\left[d^{2}\left(f\left(x_{1}, \ldots, x_{n}\right)\right), f\left(x_{1}, \ldots, x_{n}\right)\right]=0$ for all $x_{1}, \ldots, x_{n} \in \rho$ then $\rho C=e R C$ for some idempotent $e$ in the socle of $R C$ and $f\left(x_{1}, \ldots, x_{n}\right)$ is central-valued on eRCe unless $d$ is an inner derivation induced by $b \in Q$ such that $b^{2}=0$ and $b \rho=0$.

Proof. Suppose $d$ is not a $Q$-inner derivation induced by an element $b \in Q$ such that $b^{2}=0$ and $b \rho=0$.

Now assume first that $f(\rho) \rho=0$, that is $f\left(x_{1}, \ldots, x_{n}\right) x_{n+1}=0$ for all $x_{1}, x_{2}, \ldots, x_{n+1} \in \rho$. Then by [12, Proposition], $\rho C=e R C$ for some idempotent $e \in \operatorname{soc}(R C)$. Since $f(\rho) \rho=0$, we have $f(\rho R) \rho R=0$ and hence $f(\rho Q) \rho Q=0$ by [4, Theorem 2]. In particular, $f(\rho C) \rho C=0$, or equivalently, $f(e R C) e=0$. Then $f(e R C e)=0$, that is, $f\left(x_{1}, \ldots, x_{n}\right)$ is a PI for $e R C e$ and, a fortiori, central valued on $e R C e$.

Next assume that $f(\rho) \rho \neq 0$, that is $f\left(x_{1}, \ldots, x_{n}\right) x_{n+1}$ is not an identity for $\rho$ and then we derive a contradiction. By Lemma 2.3, $R$ is a GPI-ring 
and so is also $Q$ (see [1] and [4]). By [16], $Q$ is a primitive ring with $H=\operatorname{soc}(Q) \neq 0$. Moreover, we may assume $f(\rho H) \rho H \neq 0$, otherwise by [1] and [4], $f(\rho Q) \rho Q=0$, which is a contradiction. Choose $a_{0}, a_{1}, \ldots, a_{n} \in \rho H$ such that $f\left(a_{1}, \ldots, a_{n}\right) a_{0} \neq 0$. Let $a \in \rho H$. Since $H$ is a regular ring, there exists $e^{2}=e \in H$ such that

$$
e H=a H+a_{0} H+a_{1} H+\cdots+a_{n} H .
$$

Then $e \in \rho H$ and $a=e a, a_{i}=e a_{i}$ for $i=0,1, \ldots, n$. Thus we have $f(e H e)=f(e H) e \neq 0$. By our assumption and by [14, Theorem 2], we also assume that

$$
\left[d^{2}\left(f\left(x_{1}, \ldots, x_{n}\right)\right), f\left(x_{1}, \ldots, x_{n}\right)\right]
$$

is an identity for $\rho Q$. In particular $\left[d^{2}\left(f\left(x_{1}, \ldots, x_{n}\right)\right), f\left(x_{1}, \ldots, x_{n}\right)\right]$ is an identity for $\rho H$ and so for $e H$. It follows that, for all $r_{1}, \ldots, r_{n} \in H$,

$$
0=\left[d^{2}\left(f\left(e r_{1}, \ldots, e r_{n}\right)\right), f\left(e r_{1}, \ldots, e r_{n}\right)\right] .
$$

We may write $f\left(x_{1}, \ldots, x_{n}\right)=t\left(x_{1}, \ldots, x_{n-1}\right) x_{n}+h\left(x_{1}, \ldots, x_{n}\right)$, where $x_{n}$ never appears as last variable in any monomials of $h$. Let $r \in H$. Then replacing $r_{n}$ with $r(1-e)$, we have

$$
0=\left[d^{2}\left(t\left(e r_{1}, \ldots, e r_{n-1}\right) \operatorname{er}(1-e)\right), t\left(e r_{1}, \ldots, e r_{n-1}\right) \operatorname{er}(1-e)\right] .
$$

Now, we know the fact that $d(x(1-e)) e=-x(1-e) d(e)$ and $(1-e) d(e x)=$ $=(1-e) d(e) e x$ and so

$$
\begin{aligned}
(1-e) d^{2}(e x(1-e)) e & =(1-e) d\{d(e) \operatorname{ex}(1-e)+e d(e x(1-e))\} e \\
& =(1-e) d(e) d(e x(1-e)) e+(1-e) d(e) d(e x(1-e)) e \\
& =-2(1-e) d(e) \operatorname{ex}(1-e) d(e) .
\end{aligned}
$$

Thus using this facts, we have from (4),

$$
\begin{aligned}
0 & =(1-e)\left[d^{2}\left(t\left(e r_{1}, \ldots, e r_{n-1}\right) \operatorname{er}(1-e)\right), t\left(e r_{1}, \ldots, e r_{n-1}\right) \operatorname{er}(1-e)\right] \\
& =(1-e) d^{2}\left(t\left(e r_{1}, \ldots, e r_{n-1}\right) \operatorname{er}(1-e)\right) t\left(e r_{1}, \ldots, e r_{n-1}\right) \operatorname{er}(1-e) \\
& =-2(1-e) d(e) t\left(e r_{1}, \ldots, e r_{n-1}\right) \operatorname{er}(1-e) d(e) t\left(e r_{1}, \ldots, e r_{n-1}\right) \operatorname{er}(1-e) \\
& =-2\left((1-e) d(e) t\left(e r_{1}, \ldots, e r_{n-1}\right) e r\right)^{2}(1-e) .
\end{aligned}
$$

This implies

$$
0=-2\left\{(1-e) d(e) t\left(e r_{1}, \ldots, e r_{n-1}\right) e r\right\}^{3}
$$

that is

$$
0=-2\left\{(1-e) d(e) t\left(e r_{1}, \ldots, e r_{n-1}\right) e H\right\}^{3}
$$


By [6], $(1-e) d(e) t\left(e r_{1}, \ldots, e r_{n-1}\right) e H=0$ which implies

$$
(1-e) d(e) t\left(e r_{1} e, \ldots, e r_{n-1} e\right)=0 .
$$

Since $e H e$ is a simple Artinian ring and $t(e H e) \neq 0$ is invariant under the action of all inner automorphisms of $e \mathrm{He}$, by [5, Lemma 2], $(1-e) d(e)=0$ and so $d(e)=e d(e) \in e H$. Thus $d(e H) \subseteq d(e) H+e d(H) \subseteq e H \subseteq \rho H$ and $d(a)=d(e a) \in d(e H) \subseteq \rho H$. Therefore, $d(\rho H) \subseteq \rho H$. Denote the left annihilator of $\rho H$ in $H$ by $l_{H}(\rho H)$. Then $\overline{\rho H}=\frac{\rho H}{\rho H \cap l_{H}(\rho H)}$, a prime $C$-algebra with the derivation $\bar{d}$ such that $\bar{d}(\bar{x})=\overline{d(x)}$, for all $x \in \rho H$. By assumption, we have that

$$
\left[\bar{d}^{2}\left(f\left(\overline{x_{1}}, \ldots, \overline{x_{n}}\right)\right), f\left(\overline{x_{1}}, \ldots, \overline{x_{n}}\right)\right]=0
$$

for all $\overline{x_{1}}, \ldots, \overline{x_{n}} \in \overline{\rho H}$. By Theorem $A$, either $\bar{d}=0$ or $f\left(\overline{x_{1}}, \ldots, \overline{x_{n}}\right)$ is central-valued on $\overline{\rho H}$.

If $\bar{d}=0$, then $d(\rho H) \rho H=0$ and so $d(\rho) \rho=0$. By Lemma 2.1, $d$ is an inner derivation induced by an element $b \in Q$ such that $b \rho=0$. Then for all $x_{1}, \ldots, x_{n} \in \rho$, we have by assumption that

$$
0=\left[\left[b,\left[b, f\left(x_{1}, \ldots, x_{n}\right)\right]\right], f\left(x_{1}, \ldots, x_{n}\right)\right]=-f^{2}\left(x_{1}, \ldots, x_{n}\right) b^{2} .
$$

By [3, Lemma 4], either $b^{2}=0$ or $f(\rho) \rho=0$. In both cases we have contradiction.

If $f\left(\overline{x_{1}}, \ldots, \overline{x_{n}}\right)$ is central-valued on $\overline{\rho H}$, then $\rho H$, as well as $\rho$, satisfies $\left[f\left(x_{1}, \ldots, x_{n}\right), x_{n+1}\right] x_{n+2}=0$. Then $\rho C=e R C$ for some idempotent element $e \in \operatorname{soc}(R C)$ by [12, Proposition] and $f\left(x_{1}, \ldots, x_{n}\right)$ is central-valued on $e R C e$ and we are done.

THEOREM 2.5. Let $R$ be an associative prime ring of char $R \neq 2$ with center $Z(R)$ and extended centroid $C, f\left(x_{1}, \ldots, x_{n}\right)$ a nonzero multilinear polynomial over $C$ in $n$ noncommuting variables, $d$ a nonzero derivation of $R$ and $\rho$ a nonzero right ideal of $R$. If $\left[d^{2}\left(f\left(x_{1}, \ldots, x_{n}\right)\right), f\left(x_{1}, \ldots, x_{n}\right)\right] \in Z(R)$ for all $x_{1}, \ldots, x_{n} \in \rho$ then $\rho C=e R C$ for some idempotent e in the socle of $R C$ and either $f\left(x_{1}, \ldots, x_{n}\right)$ is central-valued on eRCe or eRCe satisfies $S_{4}\left(x_{1}, x_{2}, x_{3}, x_{4}\right)$ unless $d$ is an inner derivation induced by $b \in Q$ such that $b^{2}=0$ and $b \rho=0$.

Proof. Suppose $d$ is not a $Q$-inner derivation induced by an element $b \in Q$ such that $b^{2}=0$ and $b \rho=0$.

If $[f(\rho), \rho] \rho=0$, that is $\left[f\left(x_{1}, \ldots, x_{n}\right), x_{n+1}\right] x_{n+2}=0$ for all 
$x_{1}, x_{2}, \ldots, x_{n+2} \in \rho$, then by [12, Proposition], $\rho C=e R C$ for some idempotent $e \in \operatorname{soc}(R C)$ and $f\left(x_{1}, \ldots, x_{n}\right)$ is central-valued on $e R C e$.

So, assume that $[f(\rho), \rho] \rho \neq 0$, that is $\left[f\left(x_{1}, \ldots, x_{n}\right), x_{n+1}\right] x_{n+2}$ is not an identity for $\rho$ and then we derive that $e R C e$ satisfies $S_{4}$. By Lemma 2.3, $R$ is a GPI-ring and so is also $Q$ (see [1] and [4]). By [16], $Q$ is a primitive ring with $H=\operatorname{soc}(Q) \neq 0$. Moreover, we may assume $[f(\rho H), \rho H] \rho H \neq 0$, otherwise by [1] and [4], $[f(\rho Q), \rho Q] \rho Q=0$, which is a contradiction. Choose $a_{1}, \ldots, a_{n+2}, b_{1}, \ldots, b_{5} \in \rho H$ such that $\left[f\left(a_{1}, \ldots, a_{n}\right), a_{n+1}\right] a_{n+2} \neq 0$ and $S_{4}\left(b_{1}, b_{2}, b_{3}, b_{4}\right) b_{5} \neq 0$. Let $a \in \rho H$. Since $H$ is a regular ring, there exists $e^{2}=e \in H$ such that

$$
e H=a H+a_{1} H+\cdots+a_{n+2} H+b_{1} H+\cdots+b_{5} H .
$$

Then $e \in \rho H$ and $a=e a, a_{i}=e a_{i}$ for $i=1, \ldots, n+2, b_{i}=e b_{i}$ for $i=1, \ldots, 5$. Thus we have $f(e H e)=f(e H) e \neq 0$. Moreover, by [14, Theorem 2], we may also assume that

$$
\left[\left[d^{2}\left(f\left(x_{1}, \ldots, x_{n}\right)\right), f\left(x_{1}, \ldots, x_{n}\right)\right], x_{n+1}\right]
$$

is an identity for $\rho Q$. In particular, $\left[\left[d^{2}\left(f\left(x_{1}, \ldots, x_{n}\right)\right), f\left(x_{1}, \ldots, x_{n}\right)\right], x_{n+1}\right]$ is an identity for $\rho H$ and so for $e H$. It follows that, for all $r_{1}, \ldots, r_{n+1} \in H$,

$$
0=\left[\left[d^{2}\left(f\left(e r_{1}, \ldots, e r_{n}\right)\right), f\left(e r_{1}, \ldots, e r_{n}\right)\right], e r_{n+1}\right]
$$

We may write $f\left(x_{1}, \ldots, x_{n}\right)=t\left(x_{1}, \ldots, x_{n-1}\right) x_{n}+h\left(x_{1}, \ldots, x_{n}\right)$, where $x_{n}$ never appears as last variable in any monomials of $h$. Let $r \in H$. Then replacing $r_{n}$ with $r(1-e)$ and $r_{n+1}$ with $r_{n+1}(1-e)$, we have

(5) $0=\left[\left[d^{2}\left(t\left(e r_{1}, \ldots, e r_{n-1}\right) \operatorname{er}(1-e)\right), t\left(e r_{1}, \ldots, e r_{n-1}\right) \operatorname{er}(1-e)\right], e r_{n+1}(1-e)\right]$.

Now, we know the fact that $d(x(1-e)) e=-x(1-e) d(e),(1-e) d(e x)=$ $=(1-e) d(e) e x$ and $(1-e) d^{2}(e x(1-e)) e=-2(1-e) d(e) e x(1-e) d(e)$. Thus using these facts, we have from (5),

$$
\begin{aligned}
0= & {\left[\left[d^{2}\left(t\left(e r_{1}, \ldots, e r_{n-1}\right) \operatorname{er}(1-e)\right), t\left(e r_{1}, \ldots, e r_{n-1}\right) \operatorname{er}(1-e)\right], e r_{n+1}(1-e)\right] } \\
= & {\left[d^{2}\left(t\left(e r_{1}, \ldots, e r_{n-1}\right) \operatorname{er}(1-e)\right), t\left(e r_{1}, \ldots, e r_{n-1}\right) \operatorname{er}(1-e)\right] \operatorname{er}_{n+1}(1-e) } \\
& -e r_{n+1}(1-e)\left[d^{2}\left(t\left(e r_{1}, \ldots, e r_{n-1}\right) \operatorname{er}(1-e)\right), t\left(e r_{1}, \ldots, e r_{n-1}\right) \operatorname{er}(1-e)\right] \\
= & -t\left(e r_{1}, \ldots, e r_{n-1}\right) \operatorname{er}(1-e) d^{2}\left(t\left(e r_{1}, \ldots, e r_{n-1}\right) \operatorname{er}(1-e)\right) \operatorname{er}_{n+1}(1-e) \\
& -e r_{n+1}(1-e) d^{2}\left(t\left(e r_{1}, \ldots, e r_{n-1}\right) \operatorname{er}(1-e)\right) t\left(\operatorname{er}_{1}, \ldots, e r_{n-1}\right) \operatorname{er}(1-e) \\
= & t\left(e r_{1}, \ldots, e r_{n-1}\right) \operatorname{er}(1-e) d(e) t\left(e r_{1}, \ldots, e r_{n-1}\right) \operatorname{er}(1-e) d(e) r_{n+1}(1-e) \\
& +\operatorname{er}_{n+1}(1-e) d(e) t\left(e r_{1}, \ldots, e r_{n-1}\right) \operatorname{er}(1-e) d(e) t\left(e r_{1}, \ldots, e r_{n-1}\right) \operatorname{er}(1-e) .
\end{aligned}
$$


Replacing $r_{n+1}$ with $t\left(e r_{1}, \ldots, e r_{n-1}\right) e r$ in the above relation, we get

$$
2 t\left(e r_{1}, \ldots, e r_{n-1}\right) \operatorname{er}\left((1-e) d(e) t\left(e r_{1}, \ldots, e r_{n-1}\right) e r\right)^{2}(1-e)=0 .
$$

This implies

$$
2\left((1-e) d(e) t\left(e r_{1}, \ldots, e r_{n-1}\right) e r\right)^{4}=0
$$

that is

$$
2\left\{(1-e) d(e) t\left(e r_{1}, \ldots, e r_{n-1}\right) e H\right\}^{4}=0 .
$$

By [6], $(1-e) d(e) t\left(e r_{1}, \ldots, e r_{n-1}\right) e H=0$ which implies

$$
(1-e) d(e) t\left(e r_{1} e, \ldots, e r_{n-1} e\right)=0 .
$$

Since $e H e$ is a simple Artinian ring and $t(e H e) \neq 0$ is invariant under the action of all inner automorphisms of $e H e$, by [5, Lemma 2], $(1-e) d(e)=0$ and so $d(e)=e d(e) \in e H$. Thus $d(e H) \subseteq d(e) H+e d(H) \subseteq e H \subseteq \rho H$ and $d(a)=d(e a) \in d(e H) \subseteq \rho H$. Therefore, $d(\rho H) \subseteq \rho H$. Denote the left annihilator of $\rho H$ in $H$ by $l_{H}(\rho H)$. Then $\overline{\rho H}=\frac{\rho H}{\rho H \cap l_{H}(\rho H)}$, a prime $C$-algebra with the derivation $\bar{d}$ such that $\bar{d}(\bar{x})=\overline{d(x)}$, for all $x \in \rho H$. By assumption, we have that

$$
\left.\left[\left[\bar{d}^{2} f\left(\overline{x_{1}}, \ldots, \overline{x_{n}}\right), f\left(\overline{x_{1}}, \ldots, \overline{x_{n}}\right)\right], \overline{x_{n+1}}\right]\right]=0
$$

for all $\overline{x_{1}}, \ldots, \overline{x_{n}} \in \overline{\rho H}$. By Theorem 1.3, either $\bar{d}=0$ or $f\left(\overline{x_{1}}, \ldots, \overline{x_{n}}\right)$ is central-valued on $\overline{\rho H}$ or $\overline{\rho H}$ satisfies the standard identity $S_{4}\left(\overline{x_{1}}, \ldots, \overline{x_{4}}\right)$.

If $\bar{d}=0$, then as in the proof of Theorem 2.4, we have $d(\rho) \rho=0$ and hence by Lemma 2.1, $d$ is an inner derivation induced by an element $b \in Q$ such that $b \rho=0$. Thus for all $r_{1}, \ldots, r_{n} \in \rho H$,

$$
\left[d^{2}\left(f\left(r_{1}, \ldots, r_{n}\right)\right), f\left(r_{1}, \ldots, r_{n}\right)\right]=-f\left(r_{1}, \ldots, r_{n}\right)^{2} b^{2} \in C .
$$

Commuting both sides with $f\left(r_{1}, \ldots, r_{n}\right)$, we obtain $f\left(r_{1}, \ldots, r_{n}\right)^{3} b^{2}=0$. In this case by Lemma 2.2, since $b^{2} \neq 0, f(\rho H) \rho H=0$. If $f(\rho H) \rho H=0$, then $[f(\rho H), \rho H] \rho H=0$, a contradiction.

If $f\left(\overline{x_{1}}, \ldots, \overline{x_{n}}\right)$ is central-valued on $\overline{\rho H}$, then we obtain that

$$
\left[f\left(x_{1}, \ldots, x_{n}\right), x_{n+1}\right] x_{n+2}
$$

is an identity for $\rho$, a contradiction.

Finally, if $S_{4}\left(\overline{x_{1}}, \ldots, \overline{x_{4}}\right)$ is an identity for $\overline{\rho H}, S_{4}\left(x_{1}, \ldots, x_{4}\right) x_{5}$ is an identity for $\rho H$ and so for $\rho C$ and this contradicts the choices of the elements $b_{1}, \ldots, b_{5} \in \rho H$. Therefore, we conclude that in any case $\rho C$ satisfies a polynomial identity, hence by [12, Proposition], there exists an idempotent $e \in \operatorname{Soc}(R C)$ such that $\rho C=e R C$, as desired. 
Acknowledgments. The authors would like to thank the referee for his/ her valuable comments and suggestions to modify some arguments of this paper.

\section{REFERENCES}

[1] K. I. BEIDAR, Rings with generalized identities, Moscow Univ. Math. Bull., 33 (4) (1978), pp. 53-58.

[2] M. BREŠAR, One-sided ideals and derivations of prime rings, Proc. Amer. Math. Soc., 122 (4) (1994), pp. 979-983.

[3] C. M. Chang, Power central values of derivations on multilinear polynomials, Taiwanese J. Math., 7 (2) (2003), pp. 329-338.

[4] C. L. ChUANG, GPIs having coefficients in Utumi quotient rings, Proc. Amer. Math. Soc., 103 (3) (1988), pp. 723-728.

[5] C. L. ChUANG - T. K. LeE, Rings with annihilator conditions on multilinear polynomials, Chinese J. Math., 24 (2) (1996), pp. 177-185.

[6] B. Felzenszwalb, On a result of Levitzki, Canad. Math. Bull., 21 (1978), pp. 241-242.

[7] V. De FilipPis - O. M. Di Vincenzo, Posner's second theorem, multilinear polynomials and vanishing derivations, J. Aust. Math. Soc., 76 (2004), pp. 357-368.

[8] V. K. Kharchenko, Differential identity of prime rings, Algebra and Logic., 17 (1978), pp. 155-168.

[9] C. Lanski, An Engel condition with derivation, Proc. Amer. Math. Soc., 118 (3) (1993), pp. 731-734.

[10] C. Lanski, Differential identities, Lie ideals, and Posner's theorems, Pacific J. Math., 134 (2) (1988), pp. 275-297.

[11] P. H. LeE - T. K. LEE, Derivations with engel conditions on multilinear polynomials, Proc. Amer. Math. Soc., 124 (9) (1996), pp. 2625-2629.

[12] T. K. LEE, Power reduction property for generalized identities of one sided ideals, Algebra Colloquium, 3 (1996), pp. 19-24.

[13] T. K. LEE, Left annihilators characterized by GPIs, Trans. Amer. Math. Soc., 347 (1995), pp. 3159-3165.

[14] T. K. LEE, Semiprime rings with differential identities, Bull. Inst. Math. Acad. Sinica, 20 (1) (1992), pp. 27-38.

[15] U. Leron, Nil and power central valued polynomials in rings, Trans. Amer. Math. Soc., 202 (1975), pp. 97-103.

[16] W. S. Martindale III, Prime rings satisfying a generalized polynomial identity, J. Algebra, 12 (1969), pp. 576-584.

[17] E. C. Posner, Derivation in prime rings, Proc. Amer. Math. Soc., 8 (1957), pp. 1093-1100.

Manoscritto pervenuto in redazione il 16 marzo 2008. 
